\title{
Book Review: Cold Aqueous Planetary Geochemistry with FREZCHEM - From Modeling to the Search for Life at the Limits
}

\author{
Giles M. Marion, Jeffrey S. Kargel, Springer, 2008, \\ 251 pp., ISBN: 978-3-540-75678-1 (US 119.00)
}

\section{Javier Ruiz}

Received: 8 June 2008 /Published online: 24 June 2008

Through the last decades the importance of understanding the chemistry of cold aqueous environments, both on the Earth and other planetary bodies, has become increasingly clear. The FREZCHEM program, which constitutes the core of the book written by Giles Marion and Jeffrey Kargel (as clearly stated in the title), is a powerful theoretical tool to contribute to this task. Since 1994 - date of publication of the first version of FREZCHEM - almost ten versions and adaptations progressively have increased its power, versatility and capability of dealing with chemically complex scenarios. Now, the program is mature enough to justify a book.

Indeed, this book is a guide for the introduction to, and the use of, FREZCHEM (in fact, the appendix A is literally a "program guide"), but it is more. For example, the explanation of the theoretical basis for the program building constitutes an excellent review of the principles of the thermodynamics of aqueous solutions. An additional merit is a clear specification of the program limits and conditions of applicability. Therefore, the book will be of great interest for researchers on the chemistry of cold aqueous solutions, and it is an excellent guide to the use of FREZCHEM. But the book has other prominent merits.

For example, chapter 5 includes a synthesis of results obtained by previous works using FREZCHEM, which deal with a variety of topics. For Earth, they include analysis of the chemistry of aqueous saline environments (which can be standing water bodies, but also soils or sea ice), the ocean chemistry during snowball or hothouse episodes, or the evolution of freezing seawater. FREZCHEM has also been applied to study the composition and geochemical evolution of ancient waters on Mars, and to the composition of both the icy shell and internal ocean of Europa. As recognized by the authors, the results for Mars

\footnotetext{
J. Ruiz $(\bowtie)$

Departamento de Geología, Museo Nacional de Ciencias Naturales, CSIC, José Gutiérrez Abascal 2, 28006 Madrid, Spain

e-mail: jaruiz@geo.ucm.es

J. Ruiz

Centro de Biología Molecular, CSIC-Universidad Autónoma de Madrid, 28049 Cantoblanco,

Madrid, Spain
} 
have been partially overcome by recent discoveries, which imply a greater role for acidification processes than previously thought.

Marion and Kargel also enter upon key matters for astrobiology and the search of life in the Solar System. Chapter 4 offers a review on the limits for the existence of life, such we know it. This discussion is very interesting, since it is not a purely descriptive list of admissible ranges for the different parameters considered (e.g., temperature, salinity, acidity, radiation, pressure). Indeed, the reasons for the influence of these parameters are discussed. Especially useful is the discussion on pressure, which affects, for example, chemical reactions, freezing temperatures and viscosity of aqueous solutions, factors in turn affecting biologic activity. On the other hand, the final chapter is fundamentally dedicated to meditating on the future evolution of the life in the Solar System and in the Universe.

Much of the relevant places in the Solar System with astrobiological interest are characterized by cold temperatures, such as the shallow subsurface of Mars or icy crusts and internal oceans of icy bodies, which justify the potential of FREZCHEM for Astrobiology. (As pointed out by the authors, a potentially favorable habitat for Martian micro-organisms should be located within the crust, favored by relatively high temperatures, and where the lower gravity and thermal gradients of Mars would permit acceptable conditions for life at depths higher than in the Earth. But this possible habitat is obviously much more difficult to access than the cold surface or shallower subsurface). However, the authors do not discuss the results (obtained by using FREZCHEM) reviewed in chapter 5 in the framework of the limits for life reviewed in the chapter 4. This discussion could have been very interesting for a wide variety of readers, but this defect, the only one noticeable in the book, is compensated for by the excellent review on the limits of life already noted.

In summary, this book should contribute to the increasing interest in the geochemistry and biology of the cold aqueous environments of the Solar System, because it presents a first order tool for this research. 